\title{
Les écoles de dessin linéaire de la Restauration
}

Les cas des départements du Lot et de l'Yonne, 1817-1831

\section{Gérard Bodé}

\section{(2) OpenEdition}

\section{Journals}

Édition électronique

URL : https://journals.openedition.org/artefact/8870

DOI : 10.4000/artefact.8870

ISSN : 2606-9245

Éditeur :

Association Artefact. Techniques histoire et sciences humaines, Presses universitaires du Midi

Édition imprimée

Date de publication : 11 septembre 2014

Pagination : 29-41

ISBN : 978-2-271-08150-6

ISSN : 2273-0753

\section{Référence électronique}

Gérard Bodé, "Les écoles de dessin linéaire de la Restauration », Artefact [En ligne], 2 | 2014, mis en ligne le 11 mai 2021, consulté le 24 août 2021. URL : http://journals.openedition.org/artefact/8870 ; DOI : https://doi.org/10.4000/artefact.8870

\section{(c) $(1) \odot$}

Artefact, Techniques, histoire et sciences humaines est mise à disposition selon les termes de la Licence Creative Commons Attribution - Pas d'Utilisation Commerciale - Pas de Modification 4.0 International. 


\section{Les écoles de dessin linéaire de la Restauration. Les cas des départements du Lot et de l'Yonne, 1817-1831}

Gérard BODÉ ${ }^{1}$

\section{Résumé}

À partir des départements du Lot et de l'Yonne, cet article montre l'importance des configurations locales pour le développement de l'enseignement du dessin linéaire dans la première moitié du XIX ${ }^{e}$ siècle. Envisagé comme une formation élémentaire et généraliste, celui-ci anticipe parfois les sollicitations ministérielles. Les cours confiés à des instituteurs sont autant d'expériences à l'ambition pédagogique et à la pérennité très inégales, mais leur existence dans de nombreuses communes témoigne d'un intérêt partagé.

Mots-clés : cours pour ouvriers, dessin linéaire, enseignement mutuel, instruction.

\section{Abstract}

Relying on the study of Lot and Yonne, this article shows the importance of local organisations for teaching linear drawing in the first half of the nineteenth century in France. Considered as a basic and general training, it sometimes anticipated authorities' requests. Courses assigned to teachers had very unequal durability and remained fragile, but their existence in many municipalities, sometimes before legislation, reflected a shared interest.

Keywords: instruction, line drawing, monitoring teaching, workers courses. 
Quand on évoque «la formation des dessinateurs », il faut s'interroger sur le sens du terme "dessinateur» au début $\mathrm{du} \mathrm{XIX}^{\mathrm{e}}$ siècle et sur les écoles et cours qui sont censés en assurer la formation. Car, s'il est bien question d'écoles, ou de cours $^{2}$, de dessin linéaire, ces structures ne sont pas, à proprement parler, destinées à la formation de dessinateurs. Elles constituent plutôt l'une des premières étapes de ce qui deviendra, environ cinquante ans plus tard, l'enseignement technique français. Il faut en effet attendre les travaux de la commission de l'enseignement technique de 1863-1864 pour qu'une première définition soit proposée et pour que l'État commence à se préoccuper réellement de son organisation ${ }^{3}$, même si l'intervention étatique ne devient effective qu'à compter des années 1880-1890. Jusque-là, il jouait plutôt le rôle de gardien des institutions et de dispensateur de bien maigres subsides. Les véritables écoles d'enseignement technique, avec des cours pratiques donnés dans des ateliers selon le modèle instauré par les écoles d'arts et métiers ${ }^{4}$, étaient rares. De plus, l'enseignement «professionnel» était souvent prodigué dans des établissements généralistes, comme les cours spéciaux des collèges ${ }^{5}$, les écoles primaires supérieures de Guizot ou encore l'enseignement secondaire spécial de Duruy, dont l'objectif - comme dans nos cours de dessin - consiste surtout à apporter une certaine «culture technique ${ }^{6} »-$ pour utiliser une expression contemporaine à des enfants destinés à travailler dans le commerce ou l'industrie, tout comme les cours du soir visent surtout à donner à des ouvriers déjà engagés dans le monde du travail un complément de formation théorique susceptible de contribuer au développement de leur activité professionnelle. Et cette formation théorique passe ici par le dessin linéaire, une discipline à la mode sous la Restauration et la monarchie de Juillet ${ }^{7}$. La notion de « dessinateur" revêt donc ainsi une connotation toute particulière en ces premières années du xIX siècle. Si l'on ose une analogie, le dessin apparaît ici comme un équivalent du latin dans la culture humaniste des lycées.

\section{Du dessin linéaire dans des départements ruraux ?}

Cet article porte sur les départements du Lot et de l'Yonne entre 1817 et 1831. Pourquoi ces deux départements? A priori ces territoires ne devraient pas être très réceptifs au développement $\mathrm{du}$ dessin, surtout du dessin linéaire destiné à la formation des artistes, artisans ou dessinateurs professionnels, car il s'agit de départements ruraux où l'activité industrielle revêt plutôt la forme de l'ar- tisanat $\mathrm{rural}^{8}$, y compris dans les centres urbains ${ }^{9}$. Pour le Lot, rien de comparable avec certains départements voisins comme la Corrèze ou la Dordogne qui disposaient dès le début de XIX ${ }^{\mathrm{e}}$ siècle de quelques centres industriels à partir d'activités spécialisées comme la porcelaine ou la manufacture d'armes, ou encore de la Haute-Vienne où l'industrie de la céramique et de la porcelaine 
était très liée à l'art du dessin ${ }^{10}$. L'Yonne est encore dominée par l'agriculture, et notamment la viticulture, et échappe aux mutations économiques entraînées par la révolution industrielle ${ }^{11}$. Ces régions ne se caractérisent pas non plus par un fort développement de l'instruction primaire. Le Lot, situé au sud de la célèbre ligne Saint-Malo-Genève, est un département à la traîne. En 1833, l'enquête Guizot $^{12}$ le classe au $71^{\mathrm{e}}$ rang. À la même date, la situation est un peu meilleure pour l'Yonne qui dispose d'une bonne couverture en écoles primaires, surtout masculines, et connaît un taux d'alphabétisation d'environ $50 \%{ }^{13}$. Si l'on utilise les chiffres fournis par le Dictionnaire pédagogique relatifs aux conscrits sachant "au moins lire», le Lot, avec 29,6\% entre 1827 et 1829 et $30,1 \%$ entre 1831 et 1835 , se classe successivement aux $70^{\mathrm{e}}$ et $75^{\mathrm{e}}$ rang, tandis que $\mathrm{l}^{\prime}$ Yonne avec, aux mêmes dates, $50,4 \%$ et $60,3 \%$ se classe aux $33^{\mathrm{e}}$ puis $28^{\mathrm{e}}$ rang $^{14}$.

Ces départements ont été choisis en raison de la documentation disponible. En effet, les dépouillements opérés par l'équipe du Service d'histoire de l'éducation $^{15}$ dans les archives départementales ont permis de repérer des documents, certes pas très nombreux mais néanmoins assez complets, sur ces écoles ${ }^{16}$. Mêmes s'ils posent plus de questions qu'ils n'apportent de réponses, ils sont suffisants, en comparaison des sources disponibles dans d'autres archives départementales, pour pouvoir aborder ces écoles relativement peu connues ${ }^{17}$.

L'analyse des créations d'écoles de dessin avant 1835 fait apparaître deux étapes chronologiques bien déterminées, qui correspondent aussi au cadre chronologique national. La première étape, en 1817-1818, montre l'apparition d'écoles de dessin liées à l'expansion des écoles mutuelles; cette étape est bien documentée pour le département du Lot. La seconde étape, vers 1825 , se place dans le cadre du développement des «cours de géométrie appliquée aux arts » initiés par le baron Dupin. Dans les deux cas, outre le caractère spécial conféré au dessin, ces créations sont le résultat de la conjonction de la politique nationale et des initiatives locales. Dans la majorité des départements français, la période 1817-1818 est rarement documentée; il est donc très difficile de se faire une idée exacte de l'apparition et du développement de ces écoles de dessin. Ce n'est pas le cas dans le Lot où cette première étape est la plus importante avec la mention de sept cours, tandis que la seconde, 1825-1826, n'en comporte que deux. Ces premières créations précèdent les autres ouvertures d'écoles de dessin provinciales. Le cas du Lot paraît ainsi bien singulier, même s'il est impossible de dire s'il le doit à la seule conservation de ses archives ou à la présence d'une véritable volonté de développer ces écoles de dessin. 


\section{Les écoles de dessin dans l'orbite de l'école mutuelle, 1817-1818}

En 1817 et 1818, sept cours ou écoles de dessin linéaire sont fondés à Figeac, Cahors, Gourdon, Gramat et Souillac. L'écoledeFigeacestouverteenjuillet1817, l'école de Cahors en janvier 1818, celle de Gourdon en novembre 1818, celle de Gramat et les deux écoles de garçons de Souillac au cours de l'année 1818. La dernière école, l'école de filles de Souillac, date de l'année 1819. Souillac crée donc trois cours, deux pour les garçons en 1818 et un pour les filles en 1819 .

Toutes ces écoles ont le statut d'école élémentaire publique, y compris celle de Cahors hébergée au sein du collège royal. Les archives mentionnent les «fondateurs » de ces écoles: à Cahors, c'est le directeur de l'école mutuelle municipale, et, à Figeac, c'est l'instituteur créateur de l'école mutuelle de garçons. De ce fait, les enseignants sont presque tous des instituteurs. Deux cas font exception: l'école de filles de Souillac est tenue par la sœur de l'un des deux instituteurs dirigeant les écoles de dessin de garçons. Figeac connaît une évolution plus complexe: en 1822, à la suite d'une redéfinition des objectifs de l'école, l'instituteur est remplacé par le professeur de dessin $\mathrm{du}$ collège local, ancien capitaine $\mathrm{du}$ génie et architecte municipal. L'expérience ne dure qu'un an puisqu'en 1823, c'est de nouveau l'instituteur qui dirige l'école de dessin, même si l'architecte municipal continue de prodiguer des cours. Cette évolution a été voulue par le maire de la commune, qui a élaboré une structure d'enseignement diversifiée mais conflictuelle - qui sera évoquée plus bas -, ce qui explique le rappel de l'instituteur. L'instituteur de l'école de Cahors, ouverte en janvier 1818, a été formé à l'école de dessin de Figeac créée en juillet 1817.

La fréquentation des cours n'est pas connue pour toutes les écoles; néanmoins, quand ils existent, les chiffres relèvent la fréquentation mensuelle et non pas annuelle. Les effectifs de l'école de Cahors sont d'une dizaine en 1821. L'une des deux écoles de garçons de Souillac est suivie, la même année, par une vingtaine d'élèves. À Gramat, entre 1819 et 1823, de dix à vingt-huit élèves sont répertoriés. Enfin, à Figeac, pour les années 1821 à 1823, les chiffres de présence mensuelle vont de trente à dix-neuf. Cette école apparaît ainsi comme la plus fréquentée de toutes les écoles de dessin du Lot.

Quel est l'enseignement délivré dans ces écoles? Toutes affirment suivre «la méthode Francœur sous la forme de l'enseignement mutuel», sans préciser ce qu'il faut entendre par là. Dans l'une des deux écoles de dessin de garçons de Souillac, en 1820, les élèves sont répartis en trois classes. Sur les vingt-deux élèves de cette année, quatorze sont dans la première classe, qui est donc celle des débutants, et travaillent aux figures. Huit autres élèves composent la seconde classe et l'instituteur veille à «leur faire mieux connaître la direction respective que les arêtes ou les côtés de la base d'un prisme peuvent avoir sur un plan". À cette date, la troisième division ne comprend aucun élève pour l'étude du dessin. Tous ces cours ont lieu deux fois 
par semaine et, sur les vingt-deux élèves, cinq sont considérés comme excellents.

À Gramat, au mois de novembre 1819, les dix élèves "composant la huitième classe, ont successivement fait les mêmes figures depuis 1 jusqu'à 20. Les cours étant très courts, il n'a pas été possible d'en faire plusieurs divisions, n'ayant pas encore d'ailleurs de moniteurs capables d'élever les enfants des septième et sixième classes ». Dans son rapport du 28 décembre 1819, l'instituteur Pradines affirme que trois élèves "se distinguent par-dessus les autres. Ils ne se trompent jamais d'un centimètre sur les longueurs qu'ils peuvent tracer sur l'ardoise», quoiqu'ils n'aient eu «que vingt minutes de leçons par jour». À Figeac, en 1820, l'instituteur affirme suivre la même méthode que celle adoptée dans le cours de dessin du collège, ce que le sous-préfet conteste car l'instituteur ne dispose pas des instruments nécessaires pour mettre cette méthode en pratique. En 1822, la mairie réorganise les cours pour les orienter vers la topographie.

$\mathrm{D}^{\prime}$ après ces témoignages, ces "écoles de dessin» (selon la titulature des documents) sont en fait des cours de dessin prodigués par l'instituteur sous l'autorité d'un moniteur particulier à une partie des élèves de l'école mutuelle. La seule véritable école de dessin serait celle de Figeac, à partir de 1822, puisqu'elle rassemble pour un cours spécifique des élèves du collège et de l'école mutuelle. Les enseignants ne touchent pas de traitement propre à cet enseignement, puisqu'ils sont déjà enseignants à l'école mutuelle, mais des indemnités complémentaires pour cette charge de travail. Seule la commune de Figeac, en 1828, attribue un traitement à l'architecte communal pour ses activités d'enseignant.

Le cours de Figeac est celui qui se maintient le plus longtemps puisqu'il est attesté jusqu'en 1828. Les cours de Cahors, Gramat et Souillac sont mentionnés jusqu'en 1822, celui de Gourdon jusqu'en 1823. Ces cours ne semblent donc avoir fonctionné que durant quatre ou cinq ans, à l'exception de celui de Figeac qui est resté ouvert durant onze ans.

Ces créations lotoises participent à la vogue en faveur de l'enseignement mutuel déferlant sur le pays à partir de 1816 et déjà bien étudiée ${ }^{18}$. Sous l'impulsion de la Société pour l'instruction élémentaire, créée à Paris en 1815, et des sections locales qui s'implantent dans les départements, de nombreuses écoles mutuelles s'établissent dans toutes les régions, notamment dans le Sud-Ouest, en Gironde, dans la Haute-Vienne, le Tarn-et-Garonne ${ }^{19}$. Ainsi, dans le département du Lot, au cours des années 1818 et 1819 , une quinzaine de ces écoles est dénombrée à Belaye, Cahors, Cajarc, Catus, Figeac, Gignac, Gourdon, Gramat, Issendolus, Mercuès, SaintCéré et Souillac. À l'exception de Cahors et de Souillac qui accueillent aussi des filles, toutes ces écoles mutuelles sont exclusivement masculines. La plupart d'entre elles se limitent à l'instruction élémentaire du lire, écrire, compter, sauf les sept écoles citées ici qui incluent le dessin linéaire dans leur cursus. L'enseignement mutuel apparaît souvent, et pas seulement dans le Lot, comme un intitulé commun et pratique, synonyme de nouveauté par opposition à l'ancien enseignement simultané, et panacée de la lutte contre l'ignorance rurale ${ }^{20}$. 
Le contenu des leçons n'est pas vraiment fixe et diverses matières comme le dessin linéaire peuvent apparaître à côté $\mathrm{du}$ socle $\mathrm{du}$ "lire, écrire, compter». Par ailleurs, ces écoles mutuelles constituent ainsi le «moule» idéal pour ces innovations pédagogiques, le seul endroit où elles puissent être expérimentées.

S'il faut croire le Dictionnaire pédagogique de Ferdinand Buisson, l'enseignement du dessin linéaire dans les écoles mutuelles démarre avec l'expérience tentée à Libourne par l'instituteur Fréjacques dans l'établissement fondé par le duc Decazes, ministre de l'Intérieur de Louis $\mathrm{XVIII}^{21}$. Le succès de ce cours amène le ministre, par sa circulaire du 8 août 1819, à préconiser par la suite l'ouverture de cours similaires dans chaque département et notamment en adoptant la méthode et la pédagogie élaborées par Francœur ${ }^{22}$. L'expérience libournaise est généralement datée de 1818, donc postérieure à l'ouverture de l'école de Figeac au cours du mois de juillet 1817. Un exemplaire de la circulaire de Decazes a bien été retrouvé aux Archives du Lot, mais, à aucun moment, les sources lotoises ne citent l'expérience de l'école de Libourne. S'il est vrai que les rapports des années 1819 à 1822 évoquent la méthode Francœur dont trois manuels auraient été envoyés en même temps que la circulaire de Decazes pour les instituteurs de Cajarc, Figeac et Saint-Céré, aucun instituteur n'a cependant reçu l'exemplaire qui lui était destiné, les sous-préfets ayant conservé ces ouvrages dans leurs dossiers administratifs, s'il faut en croire le témoignage de l'instituteur de Figeac qui remercie le sous-préfet de lui avoir «prêté» cet ouvrage ${ }^{23}$.
Quoi qu'il en soit d'une éventuelle filiation avec Libourne, toutes les écoles lotoises semblent antérieures à l'action ministérielle. Presque toutes précèdent aussi l'école de Liancourt, ouverte en avril 1819, qui est considérée comme la première école mutuelle de dessin linéaire fondée selon les vœux de Decazes $^{24}$. Cette antériorité du département du Lot sur les créations impulsées par la volonté ministérielle semble étrange et difficile à expliquer.

La rareté des sources ne permet pas de connaître les motivations de ces fondations. On constate simplement que, dès le départ, les édiles municipaux soutiennent, voire impulsent ces innovations. Les mairies participent financièrement au fonctionnement de ces cours en votant un crédit sur le budget municipal et en payant une indemnité à l'enseignant. En contrepartie, l'État octroie une subvention, notamment pour le paiement des frais de premier établissement et l'achat des fournitures. Ces subventions nationales sont mentionnées à Gourdon et à Souillac en octobre 1818, à savoir un mois avant l'ouverture des cours de Gourdon et un mois après l'ouverture de ceux de Souillac. Dès le départ, l'implication gouvernementale est évidente. Pour Figeac, un rapport du sous-préfet du 27 août 1819 met en avant l'utilité de ces cours pour les jeunes gens se destinant aux arts mécaniques. Le texte insiste sur la seule utilité des cours, en contradiction avec la volonté, affichée depuis la fin du Premier Empire et plus encore à partir de la Restauration, d'associer l'instruction à la moralisation des couches populaires, illustrée notamment avec la création de la Société d'encouragement pour l'industrie nationale, puis celle de la Société 
pour l'amélioration de l'instruction élémentaire, qui souhaitait développer les cours du soir pour ouvriers et les écoles mutuelles qui paraissent le mieux répondre à ses objectifs. La présence de plusieurs ministres, ou futurs ministres, dont Decazes, Molé ou Gouvion Saint-Cyr, parmi ses membres explique l'impulsion gouvernementale en faveur de l'enseignement élémentaire ainsi que le soutien aux multiples formes de cours $\mathrm{du}$ soir pour ouvriers ${ }^{25}$. Les écoles de dessin participent à ce mouvement. Le dessin linéaire est considéré comme une extension de l'enseignement primaire, et en tant que tel il intègre le processus de moralisation. Ses objectifs sont donc multiples et quelque peu ambivalents: il doit certes former des artistes et des artisans pour les arts mécaniques; mais il constitue aussi l'un des éléments de la moralisation des classes populaires ${ }^{26}$. Cet aspect moralisateur ne transparaît pas dans les textes, ce qui ne signifie néanmoins pas qu'il en était absent.

Faut-il aussi voir dans la disparition des écoles lotoises, en 1822-1823, un effet de changement d'orientation politique $^{27}$ ? Les sources attribuent l'échec des écoles à des problèmes de matériel. Faute de fournitures adaptées, faute même du fameux manuel de Francœur, il était impossible de délivrer un enseignement correct malgré les bons résultats obtenus chez certains élèves. Elles évoquent aussi des conflits de personnes comme à Gramat où la négligence de l'instituteur aurait abouti à l'abandon de l'école par les élèves. Ces explications, provenant de rapports officiels, peuvent cacher les vraies raisons de l'abandon. À Figeac, l'école ne survit que parce qu'elle est passée de la fonction d'école mutuelle à celle d'un véritable cours de dessin, fondé sur la topographie et accueillant des élèves de l'école primaire et du collège communal. L'importance accordée à l'enseignement de la topographie semble singulière. Selon les sources, l'objectif est fixé par le maire en 1822: l'école de dessin est réorganisée; le professeur de dessin du collège, qui est en même temps l'architecte municipal, est chargé par délibération du conseil municipal du 14 juillet 1822 de «donner gratuitement des leçons de topographie, de montrer le dessin aux élèves pensionnaires et surveiller au collège dix jeunes gens au choix de Monsieur le Maire pris dans la classe ouvrière et qui se destinent à l'exercice d'un art qui exige des compétences sérieuses ${ }^{28} »$.

\section{Les écoles de dessin comme avatar des cours pour ouvriers, $1825-1831$}

Sur le plan national, la seconde période, qui s'ouvre vers 1825 , est généralement mieux connue ${ }^{29}$. Une circulaire du ministre de l'Intérieur du 11 décembre 1825 invite les maires à ouvrir des cours de géométrie et de mécanique appliquée. Cette circulaire prend le relais des expériences du baron Dupin. Il ne s'agit plus cette fois de prodiguer un enseignement de dessin linéaire 
à des élèves scolarisés mais d'ouvrir des cours du soir en faveur d'ouvriers ou d'apprentis déjà engagés dans le monde du travail. Si la finalité semble différente, on note un certain parallélisme: même engouement pour ces cours, même soutien de la Société pour l'instruction élémentaire, même multiplication de cours à travers tout le pays. Or, la diffusion de l'étude du dessin linéaire est citée par le baron Dupin comme un des appuis ayant permis le développement de l'étude de la géométrie et de la mécanique ${ }^{30}$. Dans les cours pour ouvriers qui connaissent le plus de succès, comme ceux de Metz, par exemple, le dessin linéaire est bien l'une des matières les mieux étudiées.

Dans le département de l'Yonne, un premier projet de "cours d'architecture et de construction» est présenté en novembre 1825 par Émile Leblanc et Tonnelier. Ce cours se divise en trois parties, les mathématiques, l'architecture et la construction. L'architecture se composera «du dessin des cinq ordres, des moulures et autres détails de décoration, tant de l'intérieur que de l'extérieur des bâtimens». Ce cours fut éphémère; un courrier du maire d'Auxerre du 19 mars 1828 qui le présentait comme "cours de dessin linéaire», signale qu'il n'était que faiblement suivi et que son initiateur «a suivi depuis une autre carrière». Le préfet de l'Yonne indique, le 26 mars 1826, qu'aucun cours de dessin linéaire n'existait dans le département. Le tableau dressé deux ans plus tard, en 1828, annonce la création imminente de cours de dessin linéaire à Auxerre et à Sens. Seule la ville de Tonnerre a ouvert, «depuis plusieurs années», un cours de dessin gratuit pour la figure, l'ornement et l'architecture. Ce cours est dirigé par l'ancien architecte municipal Joubert, «dans l'unique but de se rendre utile à ses concitoyens ». Il bénéficie d'une subvention du conseil municipal et se tient dans une salle mise à disposition par la mairie. Afin de répondre aux sollicitations du baron Dupin, la commune envisage aussi d'ouvrir «un cours pour la géométrie et la mécanique d'après le système de Mr. Dupin». Le cours de dessin linéaire accueille de douze à quinze élèves. En l'associant au cours de géométrie, la mairie espère attirer vingtcinq à trente élèves. Selon le règlement du 27 août 1828, le cours fonctionne du 2 novembre au 30 août, tous les jours de la semaine, "à l'heure de la journée qui sera le moins préjudiciable aux travaux particuliers des élèves». "Il se compose des matières suivantes: $1^{\circ}$ de la géométrie pratique, servant $\mathrm{d}$ 'introduction aux autres parties; $2^{\circ}$ de l'architecture civile avec des exemples appuyés sur les principes et appliqués aux différens genres de constructions \& décors; $3^{\circ}$ de la mécanique appliquée aux machines usuelles; $4^{\circ}$ de la perspective».

L'enseignement donné dans ce cours est décrit par l'architecte Joubert, dans une lettre au maire datée du 25 janvier $1829^{31}$ :

«Voici le mode que j'ai cru devoir employer pour le rendre profitable aux élèves. Ayant remarqué que dans quelques villes, le professeur traçait simplement à la craye sur le tableau les figures puis en donnait la démonstration d'une manière scientifique, et ayant entendu plusieurs élèves dire qu'ils n'y comprenaient rien, j'ai cru devoir adopter un mode qui, à la vérité, me donne plus de travail mais ce travail fait 
restera pour mes successeurs. Je dessine en grand et géométriquement sur de grands cartons toutes les figures chacune accompagnée de son numéro et de ses lettres et chiffres indicateurs. Je présente la figure aux élèves pour qu'ils en reconnaissent la forme, ensuite je la leur fais dessiner géométriquement. Lorsque j'ai vérifié le dessin de chacun, je leur en dicte et fais écrire la démonstration que j'ai rédigé moi-même dans des termes à leur portée. De cette manière, il doit résulter deux avantages; le premier est que chaque élève peut conserver son cours complet de figures géométriquement tracées et de démonstration de chaque figure auquel il pourra recourir en tout temps. Le second est que les quatre parties qui doivent composer ce cours tel que je l'ai annoncé, étant établies et tracées en grand sur des cartons, et ces cartons classés et distribués chaque branche dans sa caisse particulière, ainsi que les cayers de démonstration, formeront le fond qui ne laissera à mes successeurs que le travail d'expliquer et de faire exécuter. Comme je l'ai annoncé, Monsieur le Maire, toutes les figures étant numérotées, nous en avons jusqu'à ce moment parcouru et exécuté 104 et chaque élève les a sur son cayer ainsi que la démonstration. Chacun comprend ce qu'il fait et j'espère comprendra ce qu'il fera dans la suite. Comme ce cours est destiné particulièrement à la classe des jeunes ouvriers, j'ai cru devoir adopter l'heure ou ils ont quitté leur travail. C'est pourquoi, je commence à huit heures du soir. »

Le cours est encore mentionné en 1831. Il s'organise selon un cursus de deux années: la première année accueille douze élèves et la seconde quatre. Le cours prodigue à la fois des leçons de géométrie pratique, de dessin linéaire et de trigonométrie. Quant à son utilité, le témoignage du maire est réservé: "Je ne puis citer aucune preuve positive de ces avantages parce que les jeunes gens qui ont suivi le cours de son établissement ont quitté le pays pour aller, suivant l'usage, se perfectionner dans les autres villes ou sans doute ils mettent maintenant à profit les connaissances qu'ils ont acquises $^{32}$ ». L'investissement fait localement en faveur de cet enseignement ne profiterait pas à sa cité, car il favorise la mobilité de ceux qui en ont bénéficié. Pour cet édile, les cours représentent un véritable atout professionnel pour leurs auditeurs et, par extension, pour la commune. L'argumentaire de «l'utilité », déjà présente dans les documents lotois, réapparait donc ici.

La ville d'Avallon envisage l'ouverture d'un cours de dessin linéaire en mai 1828, vote un crédit et charge un professeur de mathématiques de l'assurer. En mars 1828, un instituteur communal avait déjà projeté d'ouvrir un cours, payant, car «il assure qu'il connaît aussi parfaitement que possible la méthode de $\mathrm{Mr}$. Francoeur et qu'il en a déjà fait l'application avec succès». Le cours projeté par la mairie ouvre en janvier 1829 dans une salle du collège, avec trente-neuf élèves, «tous fils d'artisans». En revanche, le conseil municipal de Sens hésite à s'engager car, selon un courrier du maire $\mathrm{du}$ 26 janvier 1829, "le conseil municipal ne fut pas alors frappé de l'utilité de ce cours » en raison de la faible industrie de la ville. À Joigny, malgré l'avis favorable du conseil municipal, le cours ne put être organisé faute d'enseignant. 
En 1831, des cours de dessin linéaire fonctionnent à Auxerre, où ils sont jumelés à un cours de géométrie et de mécanique appliquée aux arts et métiers et accueillent cent quarante-quatre élèves pour les deux cours, ainsi qu'à Avallon et à Tonnerre; en revanche Sens et Joigny n'ont pas réussi à organiser l'enseignement du dessin linéaire.

Une différence notoire avec les premiers cours lotois se mesure dans l'Yonne par l'absence complète de l'enseignement mutuel comme support des cours de dessin linéaire. Cet enseignement est très faiblement développé dans l'Yonne ${ }^{33}$. Ces cours accompagnent simplement des cours de géométrie proposés par Dupin. Pour des raisons d'organisation, les deux types de cours sont réunis à Auxerre et Tonnerre. Pour Auxerre, en 1831, ce sont bien deux enseignants différents qui sont à l'œuvre, mais dans les mêmes locaux, sous l'autorité du maire. À Tonnerre, toujours en 1831, les deux enseignements sont réunis dans un cursus de deux ans avec un seul enseignant.

Dans le département du Lot, la circulaire de 1825 donne naissance à deux projets à Cahors. Le premier, qui prévoit l'ouverture d'un cours de géométrie et de mécanique appliquée aux arts et métiers au cours de l'année 1826, émane d'un professeur de physique du collège, qui s'abrite derrière les demandes pressantes du préfet en réponse aux injonctions ministérielles. La mairie accepte de s'engager financièrement. Il est précisé que ce cours devait poursuivre l'action de l'école de dessin linéaire. Faute d'autorisation administrative, le cours ne fut jamais ouvert. Les raisons de ce refus ne sont pas argumentées. Le second projet cadurcien, celui $\mathrm{d}^{\prime}$ «école gratuite de dessin appliquée aux arts industriels ", est déposé auprès du préfet, en juillet 1826, par un particulier qui semble bien être le professeur de physique à l'origine du projet précédent et qui entend suivre l'exemple d'autres villes du Midi, non nommées dans son courrier, qui ont accordé leur protection «aux arts et aux industries ${ }^{34} »$. Cette école, ouverte au cours de l'année 1826, connaît une existence durable puisqu'elle est signalée au moins jusqu'en 1901. En 1880, elle est qualifiée $d^{\prime}$ ' école professionnelle» lors de la grande enquête nationale qui précède la promulgation de la loi sur les écoles manuelles d'apprentissage ${ }^{35}$. On ne sait pas grand-chose de l'enseignement prodigué. Un courrier d'un professeur de 1856 mentionne cependant, comme matières enseignées, la géométrie pratique, la mécanique, la coupe des bois, la taille des pierres, l'architecture, la plastique, la sculpture, la figure, l'ornement, la perspective et le paysage. Ici, la finalité professionnelle est donc affirmée. Mais, entre 1826 et 1856, c'est-à-dire pendant trente ans, les sources sont muettes. Il paraît donc bien difficile de connaître la nature de l'enseignement. 


\section{Le dessin linéaire comme base de l'enseignement technique?}

Quelle importance faut-il accorder à ces cours de dessin dans le cadre de départements ruraux au début du XIX ${ }^{e}$ siècle? Ce qui frappe d'emblée, c'est le rôle de proto-enseignement technique que peuvent revêtir ces différentes catégories de cours que l'on voit apparaître entre 1800 et 1830 dans divers départements. Quelle que soit la dénomination de ces cours, le dessin, et notamment le dessin linéaire, y apparaît presque toujours. L'une des difficultés résulte de la faible densité et de la nature des sources disponibles. Un véritable travail de recensement de tous ces cours des années 1800-1840 reste à faire dans les archives départementales pour en appréhender la diffusion et donc aussi pour juger de l'originalité du Lot et de l'Yonne.

Dans la grande enquête de 1863-1864 sur l'enseignement technique, la moitié des écoles recensées comme écoles d'enseignement technique sont des écoles de dessin avec des titulatures diverses. On peut donc considérer que le dessin linéaire apparaît ainsi comme le berceau de l'enseignement technique de premier niveau, destiné à la formation des ouvriers et des artisans, ou aux métiers du commerce et de l'industrie, pour reprendre une expression souvent utilisée.

Mais, en ce début du XIX ${ }^{\mathrm{e}}$ siècle, la nature des cours est encore bien différente de ceux des écoles professionnelles postérieures. À la différence du modèle que les écoles d'arts et métiers imposent à partir des années 1850, on n'y trouve aucun atelier. Il s'agit donc d'un enseignement essentiellement théorique, même s'il est vrai que, dans les cours de dessin linéaire, les élèves s'exercent effectivement au tracé du dessin. Les matières enseignées ne prétendent pas être adaptées à des professions déterminées mais se limitent à des notions générales, utilisables dans l'exercice de n'importe quel métier, mais que l'enseignement usuel ne délivre pas. La finalité professionnelle est pourtant bien affirmée, autant dans les cours de dessin linéaire que dans les cours de géométrie appliquée. La filiation entre ces deux types d'établissements est également consciemment affirmée par les contemporains. Le dessin linéaire de l'époque reposait d'ailleurs principalement sur la géométrie descriptive et, dans cette optique, les cours de «type Dupin» apparaissent bien comme les continuateurs de ceux des écoles mutuelles qui comprenaient un enseignement du dessin linéaire. Dans le département de l'Yonne, les deux types d'enseignement sont donnés parallèlement.

La création de cet enseignement $\mathrm{du}$ dessin résulte d'une combinaison de volontés locales et de sollicitations ministérielles assez fréquente en ces premières années du xIx siècle. Ni dans le Lot, ni dans l'Yonne, ces cours n'ont pas rencontré l'adhésion des populations visées en dépit du soutien d'une partie des élites municipales. On est loin de la réussite observée en Moselle au cours des années 1822-1830, à Mulhouse entre 1820 et $1830^{36}$, ou encore à Rouen entre 1830 et $1840^{37}$, loin aussi d'un enseignement du dessin appliqué à l'industrie ou 
d'une théorisation des rapports entre formation et productivité. Il reste cependant intéressant de noter que les archives, dans les deux départements, quel que soit l'auteur des témoignages, mettent exclusivement l'accent sur le caractère utile de ces créations. Que ce discours apparaisse chez Dupin ou chez les préfets ou sous-préfets est assez compréhensible, car ils sont les porteurs du projet. Mais on le retrouve aussi chez certains maires et instituteurs qui semblent partager cet idéal, même si l'adhésion des principaux intéressés paraît maigre. Ce qui est surtout étonnant, c'est l'absence de la rhétorique sur la moralisation ou la philanthropie, bien mise en avant par l'historiographie, et généralement bien présente dans les sources de l'époque. Le recours à d'autres types de sources s'impose pour préciser la perception de l'utilité de ce type d'enseignement.

\section{Notes}

1. Gérard Bodé est chargé d'études documentaires à l'École normale supérieure de Lyon (Institut français de l'éducation), docteur en histoire et membre du LARHRA. Il est l'auteur de L'enseignement technique de la Révolution à nos jours, tome 2: de 1927 à 1958 (2 vol.), et de trois atlas départementaux de l'enseignement technique (2004-2007). Il a récemment dirigé avec Marianne Thivend un numéro spécial d'Histoire de l'éducation ( $\mathrm{n}^{\circ} 136$, octobre-décembre 2012) sur l'enseignement commercial des filles et des garçons. Contact: gerard.bode@ens-lyon.fr.

2. Les archives emploient souvent indistinctement les termes de «cours» ou d' «écoles» pour les enseignements dont il est question ici. Ne sont concernés par ce texte que des entités postscolaires, hébergées dans des locaux qui ne leur sont pas propres, mais qui peuvent néanmoins avoir un cursus autonome. Les écoles proprement dites, comme certaines écoles de dessin fonctionnant de façon autonome, soit dans des bâtiments propres, soit au sein d'autres locaux - comme les musées de beaux-arts municipaux et délivrant un ensei- gnement exclusivement orienté vers les beauxarts (même si la notion n'est pas toujours très claire) - ne sont pas prises en compte ici.

3. Ministère de l'Agriculture, du Commerce et des Travaux publics. Commission de l'enseignement technique, Rapports et notes, Paris, Imprimerie impériale, 1865, 2 vol.

4. Charles Rodney Day, Les écoles d'arts et métiers. L'enseignement technique en France, XIX ${ }^{e}$-XXe siècle, Paris, Belin, 1991.

5. Philippe Marchand, "Une nouvelle donne sous la Restauration et la monarchie de Juillet. Les cours spéciaux des collèges dans l'académie de Douai», Histoire de l'éducation, juillet-septembre 2011, n 131, p. 5-26.

6. Charles Rodney Day, «Technical and professional education in France: the rise and fall of l'Enseignement secondaire spécial, 1865-1902», Journal of Social History, 1972-1973, vol. 6, p. 177201; François Soulard, La mise en place des formations intermédiaires en chimie appliquée au XIXe siècle: de Jean-Antoine Chaptal à Victor Duruy (1800-1869), thèse de doctorat, Université de Nantes, 2006, p. 341-375.

7. Renaud d'Enfert, L'enseignement $d u$ dessin en France: figure humaine et dessin géométrique, 1750-1850, Paris, Belin, 2003; Agnès Lahalle, Les écoles de dessin au XVIII siècle. Entre arts libéraux et arts mécaniques, Rennes, Presses universitaires de Rennes, 2006, notamment p. 30-57.

8. Jean-Claude Farcy, «L'artisanat rural en Beauce au XIX siècle», Histoire, économie et société, 1986, n 4 , p. 573-590.

9. Jean Lartigaut (dir.), Histoire $d u$ Quercy, Toulouse, Privat, 1993; Jean Richard (dir.), Histoire de la Bourgogne, Toulouse, Privat, 1978, p. 341-394

10. Alain Corbin, Archaïsme et modernité en Limousin au XIXe siècle, Limoges, Presses universitaires de Limoges, 1998; Georges Dauger, Daniel Dayen, Histoire du Limousin contemporain. Corrèze, Creuse, Haute-Vienne de 1789 à nos jours, Limoges, L. Soumy, 1997; Arlette Higounet-Nadal (dir.), Histoire du Périgord, Toulouse, Privat, 1983.

11. J. Richard (dir.), Histoire de la Bourgogne, op. cit.

12. Sur l'enquête organisée par Guizot en préparation de sa loi sur l'instruction primaire, voir le site du Service d'histoire de l'éducation: http:// www.inrp.fr/she/guizot/

13. Marie-Laurence Netter, Alphabétisation et scolarisation dans l'Yonne et la Haute-Garonne de la fin $d u$ XVII siècle à 1833 , thèse de $3^{e}$ cycle sous la dir. de François Furet, Université de Paris 1, 1980, p. 303.

14. Ferdinand Buisson, Dictionnaire pédagogique et d'instruction primaire, Paris, Hachette, 1888, part. 1, t. 2, p. 1716 (Lot) et 3003 (Yonne). 
15. Gérard Bodé, «Constitution d'un atlas-répertoire des établissements d'enseignement technique de 1789 à 1945 ", Histoire de l'éducation, n 66, mai 1995, p. 201-207.

16. Sauf exceptions qui seront signalées, les documents utilisés ici sont conservés aux Archives départementales du Lot, sous les cotes 1 T 38 et 39, et aux Archives départementales de l'Yonne, sous la cote $66 \mathrm{~T} 1$.

17. Gérard Bodé, Élisabeth Folz-Gaveau, Les établissements d'enseignement technique en France, 17891940. Tome 2: Le Lot, Lyon, INRP, 2005, p. 22-25.

18. François Jacquet-Francillon, Naissances de l'école du peuple: 1815-1870, Paris, Les Éditions de l'Atelier, 1995, p. 46-157; R. d'Enfert, L'enseignement $d u$ dessin en France..., op. cit., p. 101-118.

19. Robert-Raymond Tronchot, L'enseignement mutuel en France de 1815 à 1833: les luttes politiques et religieuses autour de la question scolaire, thèse de doctorat ès lettres, Université de Paris, 1973, t. 1, p. 454-455.

20. Michel Chalopin, L'enseignement mutuel en Bretagne. Quand les écoliers bretons faisaient la classe, Rennes, Presses universitaires de Rennes, 2011, p. 19-26.

21. Ferdinand Buisson, Dictionnaire pédagogique et d'instruction primaire, Paris, Hachette, 1887, vol. 1, p. 692-693.

22. Ibid., p. 1099-1100.

23. AD Lot, 1 T 39, lettre de l'instituteur Marie au sous-préfet de Figeac, 13 septembre 1819. Une réponse du successeur du sous-préfet de Figeac, presque dix ans après ces faits, le 24 février 1828, signale néanmoins que l'ouvrage aurait été envoyé le 7 octobre 1819, mais il peut s'agir de l'exemplaire "prêté».

24. R. d'Enfert, L'enseignement $d u$ dessin en France..., op. cit., p. 109.

25. Une analyse de la composition et de l'action de la Société d'instruction élémentaire se trouve chez F. Jacquet-Francillon, Naissances de l'école du peuple..., op. cit., p. 46-70.

26. R. d'Enfert, L'enseignement $d u$ dessin en France..., op. cit., p. 102-109.

27. Ibid., p. 116-118.

28. AD Lot, $1 \mathrm{~T} 39$.

29. Gérard Bodé, «Développement local, partenariat et alternance au $\mathrm{XIX}^{\mathrm{e}}$ siècle: les cours industriels pour ouvriers et artisans", dans
François Cardi et André Chambon (dir.), Métamorphoses de la formation. Alternance, partenariat, développement local, Paris, L'Harmattan, 1997, p. 53-74; $i d$. , "La naissance de l'enseignement technique provincial en France dans la première moitié du XIX ${ }^{e}$ siècle: étude de cas pour Metz et Nancy (18151870)», dans André Grelon et Françoise Birck (dir.), Des ingénieurs pour la Lorraine, Metz, Serpenoise, 1998, p. 71-92.

30. Carole Christen et François Vatin, Charles Dupin (1784-1873). Ingénieur, savant, économiste, pédagogue et parlementaire du Premier au Second Empire, Rennes, Presses universitaires de Rennes, 2009; François Vatin, Morale industrielle et calcul économique dans le premier XIX siècle. L'économie industrielle de Claude-Lucien Bergery (1787-1863), Paris, L'Harmattan, 2007; G. Bodé, La naissance de l'enseignement technique provincial, op. cit.; id., «L'enseignement à Metz de 1850 à $1900 »$, dans Gérard Bodé, Pierre Denis, Jacques Hennequin, Michèle Kuntz, Christiane Pignon-Feller, Gilbert Rose et Monique Sary, Metz au temps de Verlaine, Metz, Serpenoise, 1996, p. 65-76.

31. Le document est reproduit fidèlement avec son orthographe et ses fautes de grammaire.

32. AD Yonne, $66 \mathrm{~T}$ 1, lettre du maire de Tonnerre au sous-préfet, 5 mai 1831.

33. M.-L. Netter, Alphabétisation et scolarisation dans l'Yonne et la Haute-Garonne..., op. cit., p. 303, a pu calculer qu'en 1833 , l'enseignement mutuel n'était suivi que dans $4,7 \%$ des écoles. L'arrondissement le mieux pourvu était celui de Tonnerre avec $11 \%$.

34. AD Lot, E dépôt 42 (1 R 14), lettre du préfet $\mathrm{du}$ Lot Saint-Félix au maire de Cahors, transmettant la demande de création d'une école de dessin par le Sieur Pons, 25 juillet 1826 .

35. AN, F/17/11700; AM Cahors (non cotées); AD Lot, E dépôt 42, 1 R 14.

36. Raymond Oberlé, L'enseignement à Mulhouse de 1789 à 1870, Paris, Belles-Lettres, 1961; Florence Ott, La Société industrielle de Mulhouse 1826-1876. Ses membres, son action, ses réseaux, Strasbourg, Presses universitaires de Strasbourg, 1999.

37. André Dubuc, «Les cours techniques de la Société libre d'émulation de la Seine-Maritime», Cahiers d'histoire de l'enseignement (Rouen), 1973, $\mathrm{n}^{\circ} 1$, p. 65-76. 